\title{
The Expression of Epidermal Stem Cell Marker and SIRT1 in Atopic Dermatitis: A Discussion of Regenerative Potential
}

\author{
Jung-Won Shin, Hye-Ryung Choi, Kyung-Mi Nam, Seung-Hye Yang, Sung-Ae Kim ${ }^{1}$, Hyun-Jae Joe ${ }^{1}$, \\ Young-Ji Hwang ${ }^{2}$, Jung-Im Na, Chang-Hun Huh, Kyoung-Chan Park \\ Department of Dermatology, Seoul National University Bundang Hospital, Seongnam, ${ }^{1}$ Department of Dermatology, Keimyung University \\ School of Medicine, Daegu, ${ }^{2}$ Hayandream Dermatologic Clinic, Cheongju, Korea
}

Dear Editor:

Atopic dermatitis (AD) is a chronic inflammatory skin disease characterized by altered skin barrier function and immune dysregulation. Previously, we demonstrated that AD may be associated with defective basement membrane (BM) and low regenerative capacity ${ }^{1}$.

The regenerative capacity of the skin is related to the ability of the stem cells to self-renew and appropriately differentiate. To localize the interfollicular epidermal stem cells (IFESCs), p63 expression has conventionally been used, which has also been expressed in the upper layers of the epidermis, suggesting that it may not be a specific stem cell marker. Recently, we reported that combined staining of p63 and histone deacetylase 1 (HDAC1) can be a new method to distinguish IFESCs ${ }^{2}$. We showed that a p63-positive/HDAC1-negative expression pattern may be a better indicator of stem cells than p63-positive pattern alone. Meanwhile, sirtuin 1 (SIRT1) is a nicotinamide adenine dinucleotide-dependent protein with multiple roles in the process of aging and disease development ${ }^{3}$. It promotes the regeneration of tissues in the process of age-related degeneration or wound healing ${ }^{4,5}$. In the present study, we aimed to elucidate the expression of IFESC marker and SIRT1 in AD and discuss the regenerative capacity of AD. Tissue samples from 15 AD patients ( 11 males, 4 females; mean age $=17.33 \pm 9.84$ years) and 11 normal volunteers
(7 males, 4 females; mean age $=15.18 \pm 7.37$ years) were collected. The mean eczema area and severity index score of $\mathrm{AD}$ patients was $12.34 \pm 9.24$; all samples were harvested from the chronic eczematous skin lesions. This study was approved by the Institutional Review Board of Seoul National University Bundang Hospital (B-1002/ 094-301) and Keimyung University Hospital (DSMC 2014-11-039-007).

Immunohistochemical staining of p63 with HDAC1 and SIRT1 was performed. Furthermore, type IV collagen staining was performed to study the status of BM, a niche for IFESCs. After staining with DAPI, images were obtained by Confocal Laser Scanning Microscope (\#LSM710; Carl Zeiss, Jena, Germany) and analyzed using ZEN 2011 microscope software (Carl Zeiss). To evaluate the epidermal stem cells, six fields with the same size were randomly chosen in each sample, at $200 \times$ magnification. Then, the percentages of epidermal cells with a p63-positive/HDAC1negative expression pattern were calculated. To evaluate the expression of SIRT1 and type IV collagen, six circular areas were also selected, and the average area stained above a certain intensity was determined using an image analysis program (MetaMorphâ Microscopy Automation \& Image Analysis Software; Molecular Devices, Sunnyvale, CA, USA). The Kruskal-Wallis test using IBM SPSS Statistics ver. 20.0 (IBM Corp., Armonk, NY, USA) was used to

Received July 31, 2018, Revised November 15, 2018, Accepted for publication December 12, 2018

Corresponding author: Kyoung-Chan Park, Department of Dermatology, Seoul National University Bundang Hospital, 82 Gumi-ro 173 beon-gil, Bundang-gu, Seongnam 13620, Korea. Tel: 82-31-787-7311, Fax: 82-2-3675-1187, E-mail: gcpark@snu.ac.kr ORCID: https://orcid.org/0000-0002-8994-8038

This is an Open Access article distributed under the terms of the Creative Commons Attribution Non-Commercial License (http://creativecommons.org/ licenses/by-nc/4.0) which permits unrestricted non-commercial use, distribution, and reproduction in any medium, provided the original work is properly cited.

Copyright (c) The Korean Dermatological Association and The Korean Society for Investigative Dermatology 
A

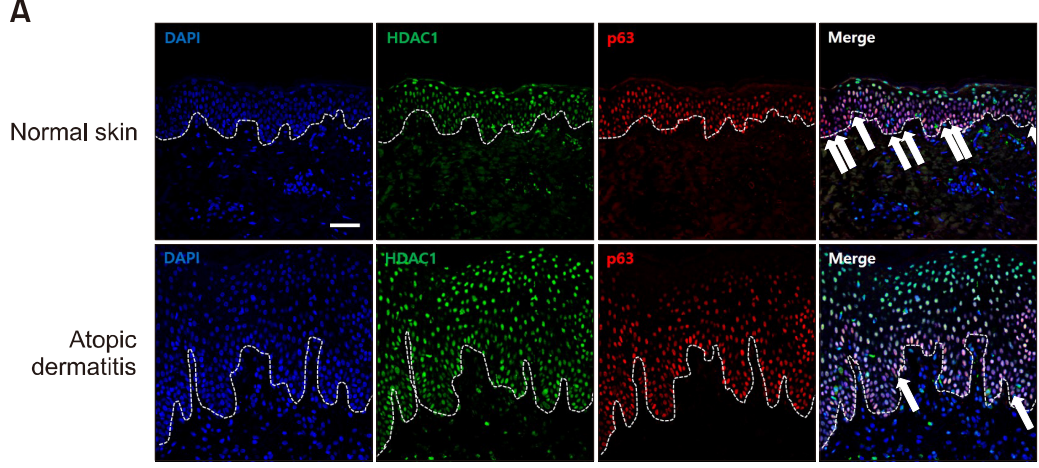

B

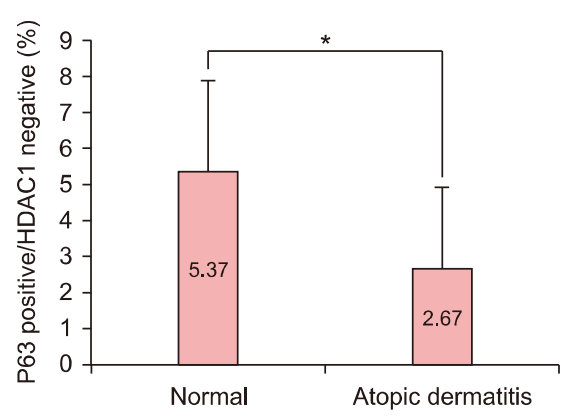

Fig. 1. Expressions of histone deacetylase 1 (HDAC1) and p63 in normal human skin and atopic dermatitis. (A) The number of p63-positive and HDAC1-negative cells (white arrows) was decreased in atopic dermatitis (green: HDAC1 staining, red: p63 staining, $\times 200$; scale bar is $50 \mu \mathrm{m}$ ). (B) The average percentages of epidermal cells with p63-positive/HDAC1-negative staining pattern were significantly lower in atopic dermatitis. SIRT1: sirtuin $1 .{ }^{*} p<0.01$.

A

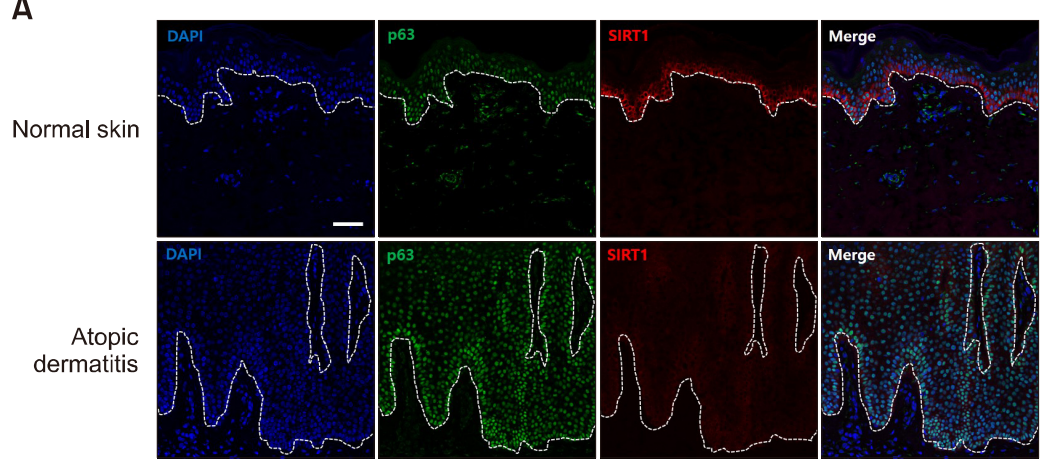

B

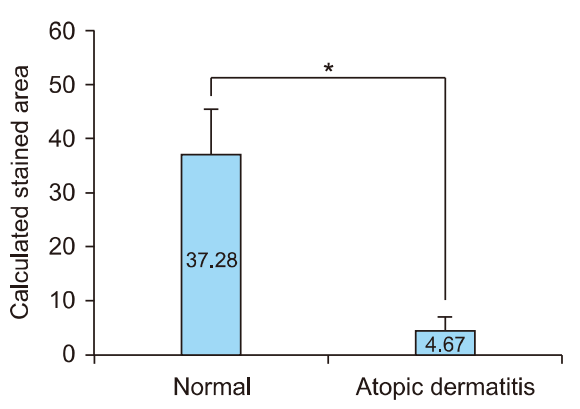

Fig. 2. Sirtuin 1 (SIRT1) expressions in normal human skin and atopic dermatitis. (A) Expression of SIRT1 was clearly reduced in atopic dermatitis (red: SIRT1, $\times 200$; scale bar is $50 \mu \mathrm{m}$ ). (B) The calculated area of immunohistochemical staining with SIRT1 was significantly smaller in atopic dermatitis. ${ }^{*} p<0.01$.

compare the expression of each marker between the two groups.

In normal skin, HDAC1 showed strong localization in the outer, differentiating cell layers, whereas p63 was most strongly expressed in the basal layer (Fig. 1A). In a merged image, there was a subpopulation showing a p63-positive/HDAC1-negative expression pattern in the basal layer (Fig. 1A). In AD skin, HDAC1 expression was different between AD skin and normal skin. HDAC1 was strongly expressed in some cells of the basal layer, and the cells with completely negative HDAC1 were rarely observed (Fig. 1A). The ratio of p63-positive to HDAC1-negative cells within the epidermis was significantly lower in AD skin compared with normal skin (Fig. 1B, $p<0.01$ ). Meanwhile, SIRT1 was clearly expressed in the basal and suprabasal layers (Fig. 2A) of normal skin. In contrast, it was not expressed in the epidermis in almost all AD cases. The calculated stained area for SIRT1 was significantly smaller in AD skin compared with normal skin (Fig. 2B, $p<0.01$ ).
The expression of type IV collagen was significantly decreased in AD skin compared with normal skin, which is similar with our previous report ${ }^{1}$. The calculated stained area was significantly smaller in AD skin compared with normal skin $(15.82 \pm 3.14$ in $A D, 25.15 \pm 5.54$ in normal, $p<0.05)$.

In the present study, our data suggests that the epidermal regenerative capacity is decreased in $\mathrm{AD}$ based on the decreased number of IFESCs in atopic skin. This might be related with the disruption of BM. The result of this study showed that type IV collagen, a main component of BM, was significantly decreased, which is consistent with our previous results ${ }^{1}$. BM is a niche for IFESCs: it is a specific microenvironment that regulates the activities and fate of the residing stem cells ${ }^{6}$. Interleukin (IL)-13, one of the cytokines known for its involvement in the development of $\mathrm{AD}$, may possibly play an important role in these defects via the stimulation of keratinocytes to produce matrix metalloproteinase (MMP) $-9^{7}$, degrading the type IV collagen, 
which is a major component of BM. Furthermore, in a previous study, IL-17-recently revealed to play a role in the pathogenesis of $A D$-significantly increased the secretion of MMP-9 in the peripheral blood mononuclear cells and polymorphonuclear cells ${ }^{8}$. Recently, BM has been reported to be involved in skin morphogenesis, homeostasis, and differentiation by controlling the dermal-epidermal junction (DEJ) interactions ${ }^{9}$. In this previous study, the skin equivalent model lacked heparan sulfate at DEJ, showing abnormal expression of filaggrin. Decreased SIRT1 expression in $\mathrm{AD}$ can be understood in the same context. As mentioned earlier, SIRT1 expression is known to be related to the regenerative potential of the skin and retina ${ }^{4,5}$. In line with this, Ming et al. ${ }^{10}$, through a mouse model, showed that SIRT1 plays a critical role in skin barrier maintenance. In that study, the SIRT1 protein level was shown to be down-regulated in human $A D$ and non-AD, which is consistent with our results. In summary, regenerative potential and barrier dysfunction are closely related with one another in AD. Further investigation, however, regarding the role of $B M$ and SIRT1 in $A D$ and non-AD is necessary.

In conclusion, our data showed that the number of IFECSs and expression of SIRT1 are decreased in AD. These findings suggest that the regenerative potential of the skin is decreased in AD. Chronic and recurrent course of $A D$ may be explained by reduced regenerative potential. Furthermore, it affords novel therapeutic target for $A D$ that focuses on the restoration of regenerative capacity.

\section{ACKNOWLEDGMENT}

This study is supported by grant number 02-2014-010 from the Seoul National University Bundang Hospital Research Fund. This study was also supported by a grant (HN14C0094) of the Korea Healthcare technology R\&D Project, Ministry of Health \& Welfare, Republic of Korea.

\section{CONFLICTS OF INTEREST}

The authors have nothing to disclose.

\section{ORCID}

Jung-Won Shin, https://orcid.org/0000-0003-1166-0189 Hye-Ryung Choi, https://orcid.org/0000-0002-7411-994X Kyung-Mi Nam, https://orcid.org/0000-0001-7696-2896
Seung-Hye Yang, https://orcid.org/0000-0002-5536-072X

Sung-Ae Kim, https://orcid.org/0000-0002-6040-6630

Hyun-Jae Joe, https://orcid.org/0000-0002-3719-4166

Young-Ji Hwang, https://orcid.org/0000-0001-5851-482X

Jung-Im Na, https://orcid.org/0000-0002-5717-2490

Chang-Hun Huh, https://orcid.org/0000-0003-3944-7777

Kyoung-Chan Park, https://orcid.org/0000-0002-8994-8038

\section{REFERENCES}

1. Shin JW, Choi YJ, Choi HR, Na JI, Kim KH, Park IA, et al. Defective basement membrane in atopic dermatitis and possible role of IL-13. J Eur Acad Dermatol Venereol 2015;29:2060-2062.

2. Shin JW, Choi HR, Nam KM, Lee HS, Kim SA, Joe HJ, et al. The co-expression pattern of p63 and HDAC1: a potential way to disclose stem cells in interfollicular epidermis. Int J Mol Sci 2017;18:E1360.

3. Imai S, Guarente L. NAD+ and sirtuins in aging and disease. Trends Cell Biol 2014;24:464-471.

4. Zeng Y, Yang K. Sirtuin 1 participates in the process of age-related retinal degeneration. Biochem Biophys Res Commun 2015;468:167-172.

5. Qiang L, Sample A, Liu H, Wu X, He YY. Epidermal SIRT1 regulates inflammation, cell migration, and wound healing. Sci Rep 2017;7:14110.

6. Boehnke K, Falkowska-Hansen B, Stark HJ, Boukamp P. Stem cells of the human epidermis and their niche: composition and function in epidermal regeneration and carcinogenesis. Carcinogenesis 2012;33:1247-1258.

7. Purwar R, Kraus M, Werfel T, Wittmann M. Modulation of keratinocyte-derived MMP-9 by IL-13: a possible role for the pathogenesis of epidermal inflammation. J Invest Dermatol 2008; 128:59-66.

8. Le Jan S, Plée J, Vallerand D, Dupont A, Delanez E, Durlach $A$, et al. Innate immune cell-produced IL-17 sustains inflammation in bullous pemphigoid. J Invest Dermatol 2014;134: 2908-2917.

9. Iriyama S, Hiruma T, Tsunenaga M, Amano S. Influence of heparan sulfate chains in proteoglycan at the dermal-epidermal junction on epidermal homeostasis. Exp Dermatol 2011;20:810-814.

10. Ming M, Zhao B, Shea CR, Shah P, Qiang L, White SR, et al. Loss of sirtuin 1 (SIRT1) disrupts skin barrier integrity and sensitizes mice to epicutaneous allergen challenge. J Allergy Clin Immunol 2015;135:936-945.e4. 